\title{
An Effective Mouse Model of Unilateral Renal Ischemia- Reperfusion Injury
}

\author{
José R. Godoy ${ }^{1}$, Grace Watson ${ }^{1}$, Carly Raspante ${ }^{1}$, Oscar Illanes ${ }^{1}$ \\ ${ }^{1}$ Department of Veterinary Biomedical Sciences, Long Island University College of Veterinary Medicine
}

\section{Corresponding Author}

José R. Godoy

Jose.Godoy@liu.edu

\section{Citation}

Godoy, J.R., Watson, G., Raspante, C., Illanes, O. An Effective Mouse Model of Unilateral Renal Ischemia-Reperfusion Injury. J. Vis. Exp. (173), e62749, doi:10.3791/62749 (2021).

\section{Date Published}

July 15,2021

\section{DOI}

$10.3791 / 62749$

URL

jove.com/video/62749

\section{Abstract}

Ischemia-reperfusion injury (IRI) is the leading cause of acute renal failure and is a significant contributor to delayed graft function. Animal models are the only available resources that mimic the complexities of the IRI-associated damage encountered in vivo. This paper describes an effective mouse model of unilateral renal IRI that delivers highly reproducible data. Ischemia is induced by occluding the right renal pedicle for $30 \mathrm{~min}$ followed by reperfusion. In addition to the surgical procedure, a sequential overview of the expected physiological and histopathological changes following renal IRI will be provided by comparing data from seven different reperfusion times $(4 \mathrm{~h}$, 8 h, 16 h, 1 day, 2 days, 4 days, and 7 days). Critical data for planning experiments ahead, such as mean surgical time, average anesthetic consumption, and body weight changes over time, will be shared. This work will help researchers implement a reliable renal IRI model and select the appropriate reperfusion time that aligns with their intended investigative goals.

\section{Introduction}

The kidneys are among the highest perfused organs in the body and are extremely susceptible to changes in blood perfusion ${ }^{1}$. Renal ischemia-reperfusion injury (IRI) remains the leading cause of acute renal failure $e^{2,3}$ and is associated with high morbidity and high mortality in hospitalized patients ${ }^{4}$. With limited therapeutic options available, ${ }^{4,5}$ renal IRI is currently the focus of several research efforts in biomedicine ${ }^{6,7}$ aiming for the development of novel therapeutic targets and the characterization of early and sensitive markers of renal injury $8,9,10$. Identifying a reliable, time and cost-effective animal model is considered essential to meet these needs. This paper presents a simple and effective mouse model of unilateral renal IRI. Ischemia is induced by clamping of the right renal pedicle for 30 $\min ^{11,12}$. A crucial part of this model is choosing the most suitable reperfusion time that will reproduce the pathological events of interest, such as tubular necrosis, polymorphonuclear inflammatory cell infiltration, or fibrosis. Therefore, researchers are provided with this sequential 
overview of representative pathological changes expected in the IRI kidney.

\section{Protocol}

The following protocol describes a survival surgery. Therefore, the highest aseptic and surgical practice is applied. All animal experiments were performed in compliance with institutional guidelines and approved by the Institutional Animal Care and Use Committee. To eliminate gender and strain-based differences in IRI effects, only male C57BL6 mice were used in the study. All animals were matched in age and weight to produce comparable results.

\section{Preparation}

NOTE: A timeline of the different experimental phases and interventions is shown in Figure 1A.

1. Clean and disinfect the surgical table before each procedure. Prepare and place all required materials (sterilized instruments and cotton swabs, sterile gauze and drapes, pre-diluted anesthetics, heating pad, sterilized vascular clamp, sterile saline solution, and skin antiseptics and suture) on the surgical table (see the Table of Materials).

2. Anesthetize male C57BL6 mice (age range 11-13 weeks) by intraperitoneal injection of ketamine/xylazine (100 mg/ $\mathrm{kg}$ and $20 \mathrm{mg} / \mathrm{kg}$ of body weight, respectively, previously diluted in sterile saline).

NOTE: Skilled animal handling is essential to minimize stress for the animal, as stress responses can negatively affect the action of anesthetics.

3. After ketamine/xylazine administration, shave the surgical area on the right flank using a razor blade and soap.
NOTE: Shaving the skin improves wound healing as well as the general outcomes of survival surgeries.

4. Disinfect the skin in the surgical area with $70 \%$ alcohol first and then with povidone iodine solution using a cotton swab.

5. After the skin preparation, place the mouse on a heating table in a ventral decubitus position and stabilize the body temperature at $37^{\circ} \mathrm{C}$ (monitored through rectal and pad sensor probes).

NOTE: Kidneys are more easily accessed and surgically exposed when placed in ventral decubitus rather than lateral.

6. While the body temperature is stabilized, apply eye ointment to the eyes of the mouse.

NOTE: Dissociative anesthetics, such as ketamine, cause the animal's eyes to remain open while anesthetized.

\section{Surgery}

1. Once the pain reflexes are absent (toe pinching with tweezers), perform an approximately $1 \mathrm{~cm}$ dorso-lateral surgical incision on the right flank using a scalpel blade. Start the incision behind the last rib and continue caudally approximately $1 \mathrm{~cm}$ parallel to the lumbar midline.

2. Transect the abdominal musculature using scissors to visualize the retroperitoneal space. Remove the small amounts of blood produced during the sectioning of the muscles using sterile cotton swabs.

NOTE: Because the dorso-lateral approach is used, the retroperitoneum, and not the peritoneal cavity, is accessed with this procedure.

3. Push the right kidney out from the abdominal cavity. Use Graefe forceps to expose the kidney carefully. 
NOTE: Always keep forceps closed to avoid traumatic injury to the kidney when placed on the abdomen and use it only to carefully push and guide the kidney towards the surgical incision and out of it.

4. Slowly expose the right kidney and identify the renal pedicle. Carefully remove the adipose tissue around the pedicle.

5. To induce ischemia, place the vascular clamp over the renal artery and vein present in the renal pedicle, avoiding clamping the adjacent ureter. Use a HalstedMosquito hemostat for manipulating the vascular clamp. NOTE: Ischemia is confirmed by the visualization of a change in color of the kidney from red-pink to dark purple (Figure 1B).

6. Cover the clamped kidney with sterile gauze soaked in saline to avoid desiccation and leave it for $30 \mathrm{~min}$.

7. Monitor anesthesia depth and humidity of the gauze periodically during this time.

NOTE: The induction dose of anesthesia is sufficient to provide analgesia until the end of the ischemic event; hence, no additional anesthetic injections are required.

8. Shortly before the end of the ischemia period, remove the gauze and uncover the kidney. Hold the HalstedMosquito hemostat, ready for clamp removal.

9. At minute 30 , open the vascular clamp with the hemostat and remove it from the renal pedicle to allow reperfusion of the kidney.

NOTE: Reperfusion is confirmed by the visualization of a change in the color of the kidney from dark purple to redpink (Figure 1C).

10. Perform the same procedures described above for sham animals without clamping of the renal pedicle.
11. After verification of the kidney color change, return the kidney to the abdominal cavity. Close the abdominal muscles with absorbable suture 5-0 using a cruciate pattern.

NOTE: A second injection of anesthetics may be required to maintain analgesia during the suturing of the muscles and the skin. Half of the initial dose has proved effective in providing analgesia until the conclusion of the surgery.

12. Close the skin with absorbable suture 5-0 using a horizontal mattress pattern. Clean the wound with a povidone iodine solution using a cotton swab.

\section{Recovery and post-surgery}

NOTE: As post-surgical time is the actual reperfusion time, proper post-surgical care is ethically mandatory and scientifically relevant. Reperfusion times can be selected as required by the researcher. Reperfusion times of 4 h, 8 h, 16 h, 1 day, 2 days, 4 days, and 7 days are compared to obtain a sequential overview of pathological changes induced by renal IRI.

1. Keep the mouse on the heating pad until it starts recovering from anesthesia.

NOTE: It is recommended to wait until the mouse begins moving its legs and attempts to move around. In cases when additional anesthetic injections are required during the surgery, the recovery time is longer. Atipamezole, an alpha-2 receptor antagonist, can be administered at a dose of $0.5 \mathrm{mg} / \mathrm{kg}$ of body weight intraperitoneally to reverse the xylazine effects and shorten the recovery phase. For pain management, buprenorphine $(0.1 \mathrm{mg} /$ $\mathrm{kg}$ of body weight, intraperitoneally) is administered pre-operatively and every 6 hours during the recovery and post-surgical phase. The use of non-steroidal anti- 
inflammatory drugs is discouraged as several drugs in this family induce nephrotoxicity and can, therefore, alter the results.

2. After recovery from anesthesia, place the mouse back in its cage with free access to water and food.

NOTE: Mashed food can be provided in a Petri dish as well as material for hiding and playing (e.g., paper sheets, paper towel tubes).

3. Monitor the mouse daily to assess wound healing, food and water intake, body weight, and behavior.

NOTE: Wound healing status was assessed using the following scale: 1 , dry; 2 , wet; 3 , partially opened; 4 , opened. Fast wound healing was documented in this study, with more than $90 \%$ of dry wounds after day 2 .

\section{Euthanasia and sample collection}

1. Euthanize the mice with sodium pentobarbital administered intraperitoneally at a dose that is twice the anesthetic dose for mice $(100 \mathrm{mg} / \mathrm{kg})$.

2. Collect fluid and tissue samples as required.

NOTE: Both kidneys, whole blood (for blood cell count), serum (for blood biochemistry), urine, the heart, and the lungs were collected. A few microliters of serum are needed for blood biochemistry analysis (blood urea nitrogen (BUN), creatinine, electrolytes). If required, 24 $\mathrm{h}$ before euthanasia, mice can be placed into metabolic cages to collect a higher urine volume that allows the determination of renal function parameters.

\section{Representative Results}

\section{Physiological parameters}

Mice recovered from this unilateral renal IRI surgery uneventfully; appeared active and alert; and showed normal eating, drinking, and behavior by the following day. Some mice may have post-IRI body weight loss, although it is usually less than $10 \%$ of the initial body weight (Figure 2). Greater body weight losses (>10\%) can be detrimental, and those animals should be removed from the study. Shamoperated mice did not show body weight changes postsurgery (measured $24 \mathrm{~h}$ after surgery). Most mice recovered their initial body weight between days 4 and 7 post-surgery (see IRI 7-day group, Figure 2). Renal function can be assessed using traditional markers such as blood urea nitrogen (BUN) and creatinine. Additionally, electrolyte levels in serum (sodium, potassium, and chloride) and an automated differential blood count were included in the analysis.

\section{Histopathological changes}

Assessment of histopathological findings was done using $4 \%$ paraformaldehyde-fixed, paraffin-embedded whole midsagittal sections of the kidney stained with hematoxylin/eosin $(\mathrm{HE})$, periodic acid Schiff, and Masson's trichrome stains. The most evident changes produced by this unilateral renal IRI model can be seen at the cortico-medullary junction, specifically in the proximal tubules, thick ascending limbs of Henle's loop, and distal convoluted tubules, as well as in the tubular interstitium (see the legend for Figure 3). Microscopic images showing the most characteristic lesions following IRI in the kidney can be seen in Figure 3. A list of the sequential histopathological findings is provided in Table 1.

A tubular injury scoring system was developed to categorize the damage over time (Figure 4). In this, five defined alterations were assessed by three different evaluators: 1) tubular epithelial attenuation; 2) brush border loss; 3) tubular necrosis; 4) luminal obstruction; and 5) presence of proteinaceous cast. An assignment of "1" indicates that the alteration is present, "0" that it is absent. 

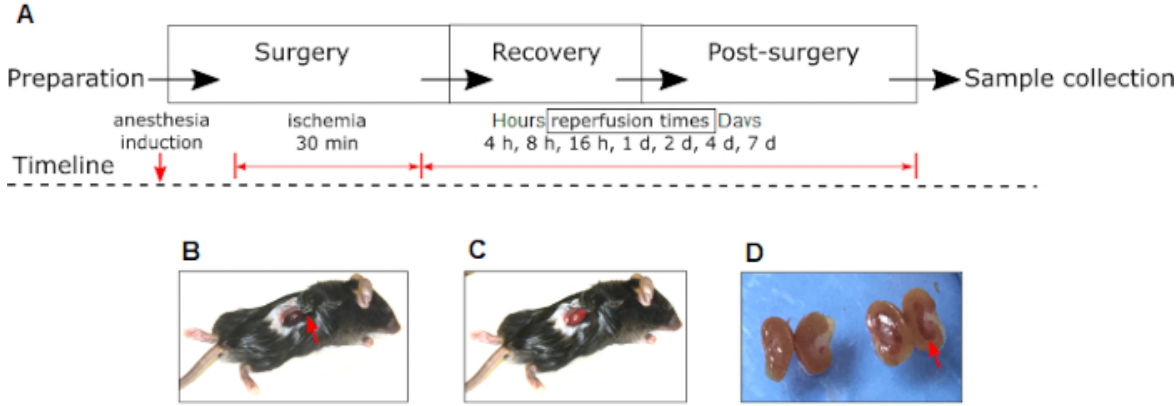

Figure 1: Experimental renal IRI model in mouse. (A) Phases of experiments and interventions (anesthesia induction, ischemia, and reperfusion) are shown. Please note the changes in the color of the right kidney to dark red during ischemia (B) to pink during reperfusion (C). (D) Macroscopic appearance of the IRI right kidney (red arrow) compared to the contralateral non-IRI kidney of the same animal $24 \mathrm{~h}$ after surgery. Red arrow in (B) shows the position of the hemostatic clamp. Abbreviation: IRI = Ischemia-reperfusion injury. Please click here to view a larger version of this figure.

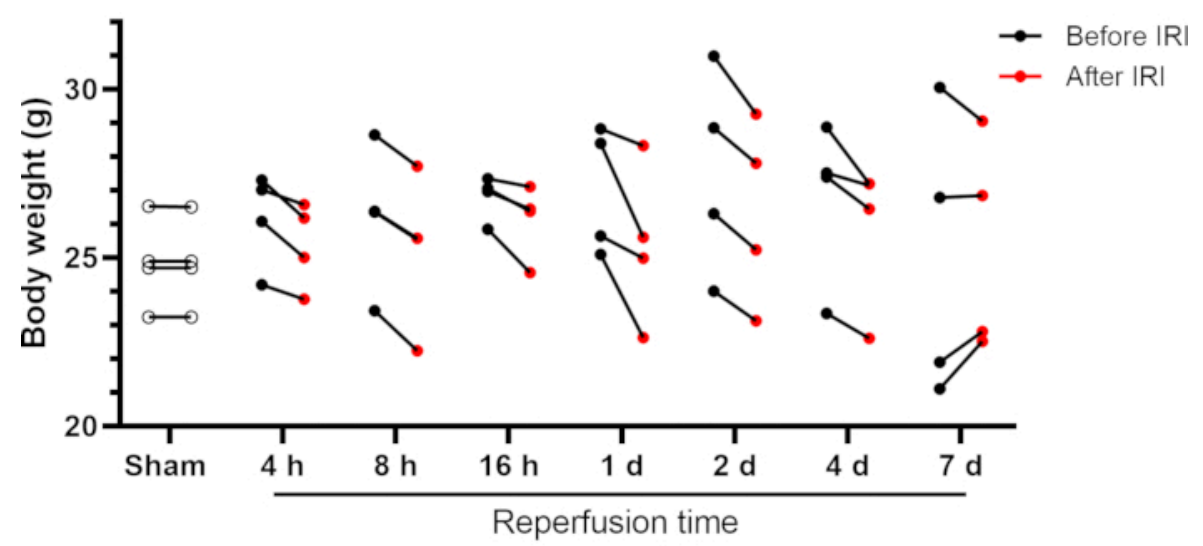

Figure 2: Body weight of mice before and after renal IRI. Individual data are shown. Abbreviations: IRI = Ischemiareperfusion injury; $\mathrm{h}=$ hours; $\mathrm{d}=$ days. Please click here to view a larger version of this figure. 
A
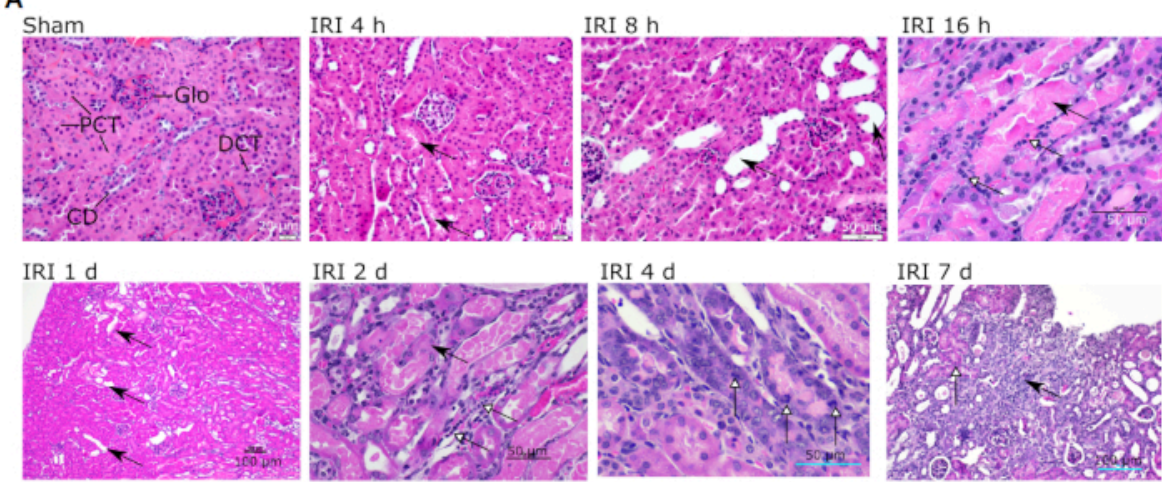

IRI $2 \mathrm{~d}$

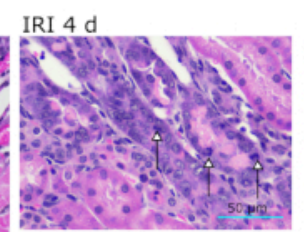

IRI $7 \mathrm{~d}$
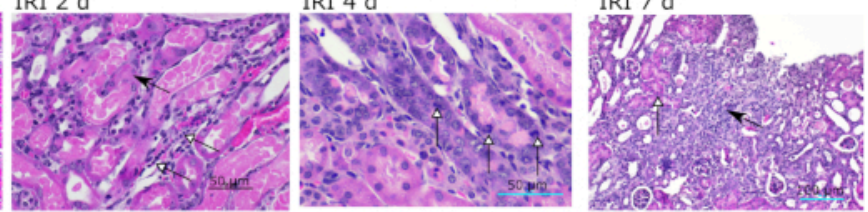

B $_{\text {Sham }}$

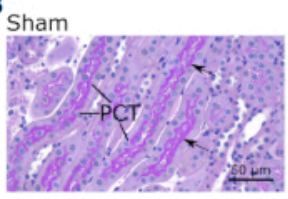

IRI $4 \mathrm{~h}$

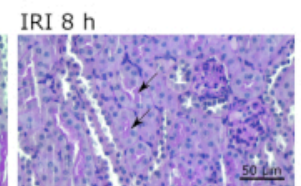

IRI $16 \mathrm{~h}$
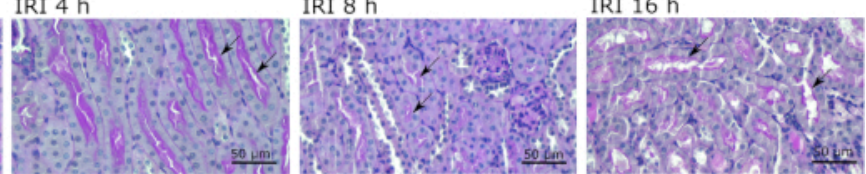

C Sham

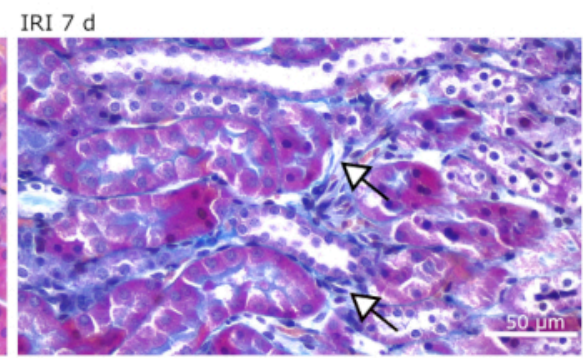

Figure 3: Typical microscopic lesions observed in the cortex and the cortico-medullary junction of IR-operated

mice. Sham and different reperfusion times are shown (indicated above each picture). (A) Intact structures are shown in sham (magnification 40x; scale bar $=20 \mu \mathrm{m}$ ). Arrows in IRI $4 \mathrm{~h}$ indicate the presence of proteinaceous cast in the tubular lumen (magnification 40x; scale bar $=20 \mu \mathrm{m}$ ). Arrows in IRI $8 \mathrm{~h}$ show tubular dilatation (magnification 40x; scale bar $=50 \mu \mathrm{m})$. Black arrow in IRI $16 \mathrm{~h}$ shows tubular cast in medullary segments; white arrows show areas of cellular necrosis (magnification 40x; scale bar $=50 \mu \mathrm{m}$ ). Black arrows in IRI $1 \mathrm{~d}$ indicate tubular dilatation (magnification 10x; scale bar $=100 \mu \mathrm{m})$. Black arrow in IRI 2 d shows enlarged cell nuclei; white arrowheads show areas of lymphocyte and macrophage infiltration (magnification 40x; scale bar $=50 \mu \mathrm{m}$ ). White arrowheads in IRI $4 \mathrm{~d}$ indicate mitotic tubular cells (magnification 40x; scale bar $=50 \mu \mathrm{m}$ ). Black arrow in IRI $7 \mathrm{~d}$ shows an area of focal fibrosis; white arrowhead shows an area of regeneration (magnification 20x; scale bar $=100 \mu \mathrm{m}$ ). (B) PAS staining showing the renal cortex of mice during early reperfusion ( $4 \mathrm{~h}, 8 \mathrm{~h}$, and $16 \mathrm{~h}$ ). Note the progressive attenuation of the brush border (arrows). Magnifications 40x; scale bars $=50 \mu \mathrm{m}$ (C) Masson trichrome staining of sham and IRI $7 \mathrm{~d}$ mice showing areas of interstitial fibrosis (white arrows). Magnification 40x; scale bars = $50 \mu \mathrm{m}$. Abbreviations: IRI = Ischemia-reperfusion injury; Glo = glomerulus; PCT = proximal 
convoluted tubule; $\mathrm{DCT}=$ distal convoluted tubule; $\mathrm{CD}=$ collecting duct; $\mathrm{PAS}=$ periodic acid Schiff; $\mathrm{d}=$ day. Please click here to view a larger version of this figure.

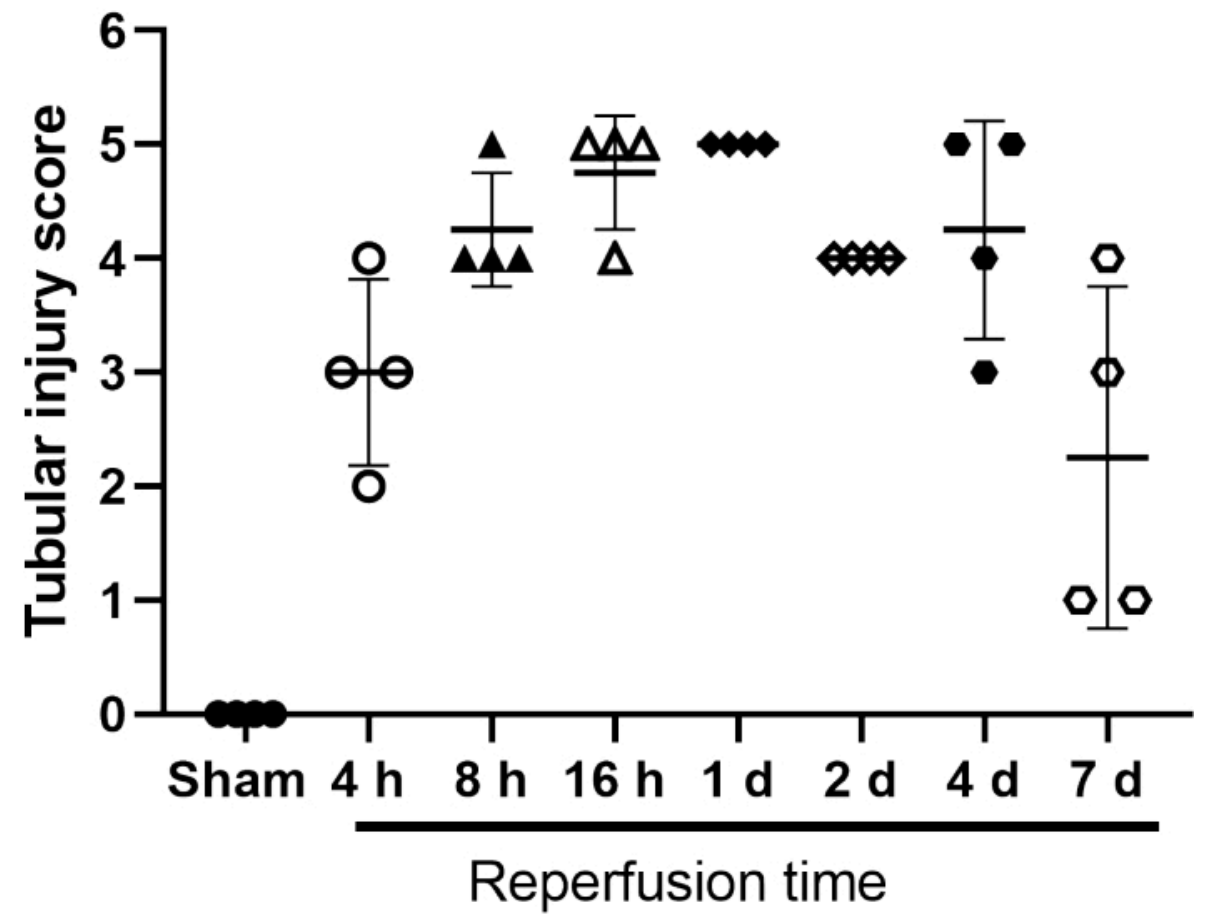

Figure 4: Tubular injury score of sham- and IRI-operated mice. Scoring system scale 1 to 5 for tubular epithelial attenuation; brush border loss; tubular necrosis; luminal obstruction; and presence of proteinaceous cast. An assignment of "1" indicates that the alteration is present, " 0 " that it is absent. Individual values are shown. Bars represent mean \pm SD $(n=$ 4). Abbreviation: IRI = Ischemia-reperfusion injury. Please click here to view a larger version of this figure. 


\begin{tabular}{|c|c|}
\hline Time after IRI & Most significant pathological changes \\
\hline \multirow[t]{2}{*}{$4 \mathrm{~h}$} & Tubular obstruction \\
\hline & Protein cast in lumen \\
\hline \multirow[t]{3}{*}{$8 \mathrm{~h}$} & Tubular dilatation \\
\hline & Incipient necrosis \\
\hline & Attenuation of epithelium \\
\hline \multirow[t]{3}{*}{$16 \mathrm{~h}$} & Cellular necrosis \\
\hline & Tubular cast \\
\hline & Neutrophil infiltration \\
\hline \multirow[t]{3}{*}{1 day } & Necrosis \\
\hline & Tubular dilatation \\
\hline & Neutrophil infiltration \\
\hline \multirow[t]{3}{*}{2 days } & Tubular dilatation \\
\hline & Lymphocyte and macrophage infiltration \\
\hline & Enlarged cell nuclei \\
\hline 4 days & Prominent mitotic activity in tubule cells \\
\hline \multirow[t]{2}{*}{7 days } & Focal fibrosis \\
\hline & Areas of regeneration \\
\hline
\end{tabular}

Table 1: Most significant pathological changes over time. Diagnosed based on microscopic examination of 4-6 animals per group.

\section{Discussion}

Mouse renal IRI models are popular in biomedical research due to their relatively low operational costs and the availability of diverse transgenic models ${ }^{12}$. The unilateral renal IRI model presented here mimics characteristic pathological changes observed in human renal IRI such as tubular dilatation, necrosis, and fibrosis ${ }^{13}$. These results are based on varying reperfusion times.
Critical steps of this protocol include the maintenance of constant body temperature and correct placement of the vascular clamp in the renal pedicle. Body temperature influences the animal's metabolism ${ }^{14}$, altering the experimental results both at the physiological and cellular levels ${ }^{15}$. In this model, the body temperature was stabilized before surgery using rectal and pad sensor probes. In addition, continuous monitoring of the body temperature 
during the whole surgical procedure is highly recommended, especially before placing the vascular clamp to induce ischemia.

The exposure of the kidney and the proper placement of the vascular clamp are also critical for the success of the experiment. Damage to the renal capsule by improper handling of the forceps during exposure of the kidney through the surgical incision will result in perirenal hemorrhage and inflammation. The vascular clamp should be placed on the renal pedicle occluding the renal artery and the renal vein without affecting the ureter and the suprarenal arteries. Critical for this step is the careful dissection of the adipose tissue surrounding the renal hilum ${ }^{14,16}$.

This model is cost- and time-effective. Anesthetic consumption per mouse was $156.47 \pm 37.88 \mu \mathrm{L}$ (mean $\pm \mathrm{SD}, \mathrm{n}=17$ ) of a prediluted ketamine/xylazine cocktail (1:10 ketamine, 1:50 xylazine, in saline; stock solution concentration, $100 \mathrm{mg} / \mathrm{mL}$ both). Surgery can be performed in a relatively short period. The total surgery time per mouse was $53 \pm 5.23 \min ($ mean $\pm S D, n=17)$. With trained personnel, several surgeries can be performed at the same time. In our group, one experienced researcher performed the surgery until the clamp was released from the renal pedicle, while a second one took over from wound closing until the recovery of the mouse. With this approach, we were able to perform a high number of surgeries on a single day. In this model, we used the dorsolateral approach, which results in less trauma and reduced fluid and heat loss from the abdominal cavity as compared with the midline approach ${ }^{16}$

Previously published protocols have described the renal pedicle clamping technique to induce acute kidney injury in mice $17,18,19$. However, in those studies, a contralateral nephrectomy was performed in addition to the unilateral IRI with ischemic times ranging from 15 to $26 \mathrm{~min}$. In this protocol, we induced unilateral ischemia for $30 \mathrm{~min}$ while preserving the contralateral kidney. This resulted in a survival rate of $100 \%$. However, this model is not suitable to induce azotemic renal damage due partly to the compensatory effect exerted by the non-surgically intervened contralateral kidney. However, keeping one kidney unaffected in the same animal offers the advantage of using longer ischemia times with a higher survival rate. In addition to this, the contralateral kidney can be utilized to assess possible side effects of test drugs or treatments applied during the experimental procedure and to study kidney-kidney crosstalk effects ${ }^{20,21}$. For example, this model has been useful in showing reactive oxygen speciesinduced alterations at the cellular level both in the IRI and contralateral, non-surgically intervened kidney ${ }^{11}$.

This model has a potential application in studies aiming to identify and characterize markers of unilateral renal damage, renal crosstalk effects, post-renal IRI-induced hemodynamic changes, and potential nephrotoxic effects of drug candidates to be used in renal IRI. This detailed description of the main pathological changes serves as a valuable tool to select the most suitable time to study specific cellular processes, from inflammation and necrosis ( $4 \mathrm{~h}$ to 2 days) to regeneration (4 days) and fibrosis (7 days and later).

\section{Disclosures}

The authors declare that there are no conflicts of interest regarding this article.

\section{Acknowledgments}

A part of the work shown in this article was founded by the Center of Integrative Mammalian Research of Ross University School of Veterinary Medicine (RUSVM), Saint Kitts and Nevis. The financial aid provided by the Department 
of Veterinary Biomedical Sciences of Long Island University College of Veterinary Medicine is greatly appreciated.

\section{References}

1. Ray, S. C., Mason, J., O'Connor, P. M. Ischemic renal injury: can renal anatomy and associated vascular congestion explain why the medulla and not the cortex is where the trouble starts? Seminars in Nephrology. 39 (6), 520-529 (2019).

2. Weight, S. C., Bell, P. R., Nicholson, M. L. Renal ischaemia--reperfusion injury. The British Journal of Surgery. 83 (2), 162-170 (1996).

3. Ratliff, B. B., Abdulmahdi, W., Pawar, R., Wolin, M. S. Oxidant mechanisms in renal injury and disease. Antioxidants \& Redox Signaling. 25 (3), 119-146 (2016).

4. Schrier, R. W., Wang, W., Poole, B., Mitra, A. Acute renal failure: definitions, diagnosis, pathogenesis, and therapy. The Journal of Clinical Investigation. 114 (1), 5-14 (2004).

5. Fernández, A. R., Sánchez-Tarjuelo, R., Cravedi, P., Ochando, J., López-Hoyos, M. Review: Ischemia reperfusion injury-a translational perspective in organ transplantation. International Journal of Molecular Sciences. 21 (22), 8549 (2020).

6. Wu, C.-L. et al. Tubular peroxiredoxin 3 as a predictor of renal recovery from acute tubular necrosis in patients with chronic kidney disease. Scientific Reports. 7 (1), 43589 (2017).

7. Nishida, K. et al. Systemic and sustained thioredoxin analogue prevents acute kidney injury and its-associated distant organ damage in renal ischemia reperfusion injury mice. Scientific Reports. 10 (1), 20635 (2020).
8. Mishra, J. et al. Neutrophil gelatinase-associated lipocalin (NGAL) as a biomarker for acute renal injury after cardiac surgery. Lancet. 365 (9466), 1231-1238 (2005).

9. Han, W. K., Bailly, V., Abichandani, R., Thadhani, R., Bonventre, J. V. Kidney injury molecule-1 (KIM-1): A novel biomarker for human renal proximal tubule injury. Kidney International. 62 (1), 237-244 (2002).

10. Coca, S. G. Kidney injury biomarkers with clinical utility: has Godot finally arrived? American Journal of Nephrology. 50 (5), 357-360 (2019).

11. Godoy, J. R. et al. Segment-specific overexpression of redoxins after renal ischemia and reperfusion: protective roles of glutaredoxin 2, peroxiredoxin 3, and peroxiredoxin 6. Free Radical Biology \& Medicine. 51 (2), 552-561 (2011).

12. Wei, Q., Dong, Z. Mouse model of ischemic acute kidney injury: technical notes and tricks. American Journal of Physiology - Renal Physiology. 303 (11), F1487-F1494 (2012).

13. Gaut, J. P., Liapis, H. Acute kidney injury pathology and pathophysiology: a retrospective review. Clinical Kidney Journal. 14 (2), 526-536 (2021).

14. Le Clef, N., Verhulst, A., D'Haese, P. C., Vervaet, B. A. Unilateral renal ischemia-reperfusion as a robust model for acute to chronic kidney injury in mice. PLoS One. 11 (3), e0152153 (2016).

15. Pelkey, T. J. et al. Minimal physiologic temperature variations during renal ischemia alter functional and morphologic outcome. Journal of Vascular Surgery. 15 (4), 619-625 (1992). 
16. Kennedy, S. E., Erlich, J. H. Murine renal ischaemiareperfusion injury. Nephrology. 13 (5), 390-396 (2008).

17. Skrypnyk, N. I., Harris, R. C., de Caestecker, M. P. Ischemia-reperfusion model of acute kidney injury and post injury fibrosis in mice. Journal of Visualized Experiments: JoVE. (78), 50495 (2013).

18. Hesketh, E. E. et al. Renal ischaemia reperfusion injury: a mouse model of injury and regeneration. Journal of Visualized Experiments: JoVE. (88), 51816 (2014).

19. Wei, J. et al. New mouse model of chronic kidney disease transitioned from ischemic acute kidney injury. American Journal of Physiology. Renal Physiology. 317 (2), F286F295 (2019).

20. Basile, D. P., Leonard, E. C., Tonade, D., Friedrich, J. L., Goenka, S. Distinct effects on long-term function of injured and contralateral kidneys following unilateral renal ischemia-reperfusion. American Journal of Physiology - Renal Physiology. 302 (5), F625-F635 (2012).

21. Polichnowski, A. J. et al. Pathophysiology of unilateral ischemia-reperfusion injury: importance of renal counterbalance and implications for the AKICKD transition. American Journal of Physiology. Renal Physiology. 318 (5), F1086-F1099 (2020). 